\title{
An Optical Sensing Material for Trace Analysis of Oxygen. Metalloporphyrin Dispersed in Poly(1-trimethylsilyl-1-propyne) Film
}

\author{
Yutaka AmaO,${ }^{\dagger}$ Ichiro OKURA, ${ }^{*}$ Hiromi ShInOHARA, ${ }^{* *}$ and Hiroyuki NiSHIDE ${ }^{* *}$ \\ Department of Applied Chemistry, Oita University, Dannoharu, Oita 870-119, Japan \\ * Department of Bioengineering, Tokyo Institute of Technology, Nagatsuta, Midori-ku, Yokohama 226-8501, Japan \\ ** Department of Polymer Chemistry, Waseda University, Okubo, Shinjuku, Tokyo 169-8555, Japan
}

(Received August 20, 2001; Accepted May 1, 2002)

\begin{abstract}
A highly gas permeable polymer, poly(1-trimethylsilyl-1-propyne) (poly(TMSP)), was applied as a matrix of the optical oxygen sensor using the oxygen-induced luminescence quenching of octaethylporphyrin (OEP) platinum (PtOEP) or palladium complex (PdOEP). OEP complex was homogeneously dispersed in poly(TMSP) to give a mechanical tough film with thickness of $10 \mu \mathrm{m}$. The luminescence intensity of OEP complex dispersed in the poly(TMSP) films drastically decreased with an increase in oxygen concentration. The oxygen sensitivity of the film $\left(I_{0} / I_{100}\right)$ was very high and estimated to be 225 for PtOEP and 121 for PdOEP, respectively. The Stern-Volmer constants of PtOEP and PdOEP dispersed in poly(TMSP) films are estimated to be 6.6 and $17 \%^{-1}$, respectively. These results indicate that OEP metal complex dispersed in the poly(TMSP) films are novel optical sensing material for trace analysis of oxygen.

KEY WORDS Poly(1-trimethylsilyl-1-propyne) / Optical Oxygen Sensor / Metalloporphyrin / Luminescence Quenching / Trace Analysis for Oxygen /
\end{abstract}

The measurement of oxygen concentration is important in various fields of chemical, clinical analysis and environmental monitoring. ${ }^{1-3}$ The sensing system for oxygen concentration is classified into titration, ${ }^{4}$ amperometry, ${ }^{5}$ chemiluminescence, ${ }^{6}$ thermoluminescence $^{7}$ and colorinmetry. ${ }^{8}$ Among these systems, the most popular method is the amperometric method using an oxygen electrode, ${ }^{5}$ in which the rate of oxygen diffusion to the cathode is measured. This system, however, is limited because of the stability of the electrode surface. Recently, a variety of devices and sensors based on luminescence quenching of organic dyes were developed to measure oxygen concentrations or partial pressures. Many studies on optical oxygen sensors are used organic dyes, such as polycyclic aromatic hydrocarbons (pyrene and its derivatives, quinoline and phenanthrene), ${ }^{9-15}$ transition metal complexes (ruthenium, ${ }^{16-21}$ osmium $^{22}$ or rhenium-polypyridine complexes ${ }^{23}$ ), and metalloporphyrins, ${ }^{24-26}$ dispersed in polymers (silicone polymer, polystyrene, etc.). Among these organic dyes, platinum and palladium porphyrins show strong phosphorescence at room temperature. ${ }^{27}$ Especially, platinum and palladium octaethylporphyrin (PtOEP and PdOEP) display strong room-temperature phosphorescence with high quantum yield $\left(\phi_{\mathrm{P}}<0.5\right)$ and long lifetime ( $c a .100 \mu \mathrm{s}$ for PtOEP and $c a .770 \mu \mathrm{s}$ for PdOEP) ${ }^{27}$ Some optical oxygen sensors based on the phosphorescence quenching of PtOEP or PdOEP have been developed by dispersing it in polymer

${ }^{\dagger}$ To whom correspendence should be addressed. films. ${ }^{28,29}$ As the organic dyes are surrounded with polymer molecules, the optical sensing performance strongly depends on the properties of polymer matrices. An oxygen permeable polymer with a low diffusion barrier for oxygen is desired. Poly(dimethylsiloxane)s have a high gas permeability but lack the mechanical strength in thin-films. On the other hand, it has recently come to be known that gas permeability of poly(1trimethylsily-1-propyne) (poly(TMSP)) film is about 10 times larger than that of poly(dimethylsiloxane) film (poly(DMS)), ${ }^{30,31}$ and provides a tough and thin film. The poly(TMSP) film is porous film with the large oxygen permeability and diffusion, indicating that the organic dye in poly(TMSP) film may contact with the oxygen in the gas phase. Thus, a poly(TMSP) film is one of the candidates for the polymer matrix of optical oxygen sensor.

In this paper, poly(TMSP) film was applied as a polymer matrix of optical oxygen sensor based on the luminescence change of PtOEP and PdOEP and the excellent oxygen sensing properties of PtOEP and PdOEP dispersed in the poly(TMSP) films are reported.

\section{EXPERIMENTAL}

\section{Materials}

PtOEP was obtained from Porphyrin Products (Logan, UT, USA). Octaethylporphyrin (OEP) was purchased from Tokyo Chemical Industry Co., Ltd. (Tokyo Japan). Polystyrene (average MW 280000, GPC grade) 
and poly(DMS) (average MW 95000, GPC grade) were purchased from Aldrich (Milwaukee, WI, USA).

\section{Synthesis of Poly(TMSP)}

Poly(TMSP) was synthesized according to the method of literature. ${ }^{31}$ 1-Trimethylsilyl-1-propyne (Chisso Co., Ltd.) $(10 \mathrm{~mL}, 68 \mathrm{mmol})$ and $\mathrm{TaCl}_{5}(0.5 \mathrm{~g}$, $1.4 \mathrm{mmol}$ ) as the catalyst were dissolved in toluene $(30 \mathrm{~mL})$, and warmed at $80^{\circ} \mathrm{C}$ for $2 \mathrm{~h}$ under nitrogen. The reaction mixture was poured into methanol and then the precipitated polymer was dissolved in toluene. The polymer was reprecipitated from the toluene solution into methanol. The obtained polymer was further purified by chromatography on polystyrene gel with toluene eluent (white powder yield 86\%). Molecular weight, polydispersity ratio, and glass transition temperature of the polymer were $8.2 \times 10^{5}, 2.3$ and $>200^{\circ} \mathrm{C}$, determined by gel permeation chromatography with tetrahydrofuran as the solvent and polystyrene as the standard and differential scanning calorimetry, respectively.

\section{Synthesis of Palladium Octaethylporphyrin}

Palladium octaethylporphyrin (PdOEP) was synthesized with refluxing OEP and excess palladium chloride in $N, N$-dimethylformamide solution at $150^{\circ} \mathrm{C}$ for $3 \mathrm{~h}$. The reaction was monitored using UV-vis spectroscopy. During synthesis of PdOEP the initial absorption bands at 400 (Soret band), 498, 530, 568, and $622 \mathrm{~nm}$ ( $\mathrm{Q}$ band) were shifted and disappeared and new bands appeared at 393 (Soret band), 512, and $546 \mathrm{~nm}$ ( $\mathrm{Q}$ band). After the mixture was cooled to room temperature, PdOEP was precipitated in water. PdOEP was collected by filtration and washed with water. The purification was performed by column chromatography on Silica gel (eluent: chloroform), followed by recrystallization.

\section{Preparation of Oxygen Sensing Film}

OEP metal complex dispersed in the poly(TMSP) film was formed by casting the mixture of $5 \mathrm{wt} \%$ poly(TMSP) and OEP metal complex in toluene onto $1.4 \times 5.0 \mathrm{~cm}$ non-luminescent glass slides. The OEP metal complex concentration in the film was approximately $2.9 \times 10^{-5} \mathrm{~mol} \mathrm{dm}^{-3}$. As the references, OEP metal complex dispersed in polystyrene film and in poly(DMS) were prepared. The films were dried at room temperature and stored in dark prior to use. The thickness of the films was determined by use of a micron-sensitive caliper. The thickness of the film prepared was $10 \mu \mathrm{m}$.

\section{Spectroscopic Measurements}

The absorption spectra of OEP metal complex dispersed in the poly(TMSP) films were recorded using a Shimadzu (Tokyo, Japan) UV-2400PC spectrometer. Steady state luminescence spectra and excitation spectra of the films were measured using a Shimadzu (Tokyo, Japan) RF-5300PC spectrofluorophotometer with a $150 \mathrm{~W}$ xenon lamp as an excitation light source. The excitation and emission bandpasses were $5.0 \mathrm{~nm}$.

Oxygen Sensing Properties of OEP Metal Complex Dispersed in the Poly(TMSP) Film

Oxygen sensing properties of OEP metal complex dispersed in the poly(TMSP) film were investigated by the oxygen-induced luminescence spectrum and intensity changes using a spectrofluorophotometer with a $150 \mathrm{~W}$ xenon lamp as an excitation light source. The sample films were mounted at a $45^{\circ}$ angle in the quartz cell to minimize light scattering from the sample and substrate. Different oxygen standards (in the range 0$100 \%$ ) in a gas stream were produced by controlling the flow rates of oxygen and argon gases entering a mixing chamber. The total pressure was maintained at 760 Torr $(1$ Torr $=133.322 \mathrm{~Pa}){ }^{28,29}$ All the experiments were carried out at room temperature. The oxygen sensing properties of OEP metal complex dispersed in the poly(TMSP) film were characterized by the $I_{0} / I_{100}$ value, where $I_{0}$ and $I_{100}$ represent the detected luminescence intensities from a film exposed to $100 \%$ argon and $100 \%$ oxygen, respectively, and the Stern-Volmer quenching constant, $K_{\mathrm{SV}}$, obtained from the following equation.

$$
I_{0} / I=1+K_{\mathrm{SV}}\left[\mathrm{O}_{2}\right]
$$

where $I_{0}, I$ and $\left[\mathrm{O}_{2}\right]$ are the luminescence intensities in the absence and presence of oxygen and oxygen concentration, respectively. The oxygen concentration $\left[\mathrm{O}_{2}\right]$ obtained from the following equation.

$$
\left[\mathrm{O}_{2}\right]=\mathrm{S}_{\mathrm{O} 2} \times p \mathrm{O}_{2}
$$

here, $S_{\mathrm{O} 2}$ and $p \mathrm{O}_{2}$ are the oxygen solubility to poly(TMSP) film and the oxygen partial pressure in the gas phase, respectively. As the $S_{\mathrm{O} 2}$ is constant value $\left(170 \mathrm{~cm}^{3}(\mathrm{STP}) \times 10^{4} \mathrm{~cm}^{-3} \mathrm{cmHg}^{-1}\right),{ }^{30}$ the $\left[\mathrm{O}_{2}\right]$ is proportional to the $p \mathrm{O}_{2}$ in the gas phase. The $K_{\mathrm{SV}}$ value was obtained from a linear plot of $\left(I_{0} / I\right)-1$ vs. $\left[\mathrm{O}_{2}\right]$.

\section{RESULTS AND DISCUSSION}

Spectroscopic Properties of OEP Metal Complex Dispersed in the Poly(TMSP) Film

The absorption spectrum of PtOEP in the poly(TMSP) film was almost the same as in a solution 

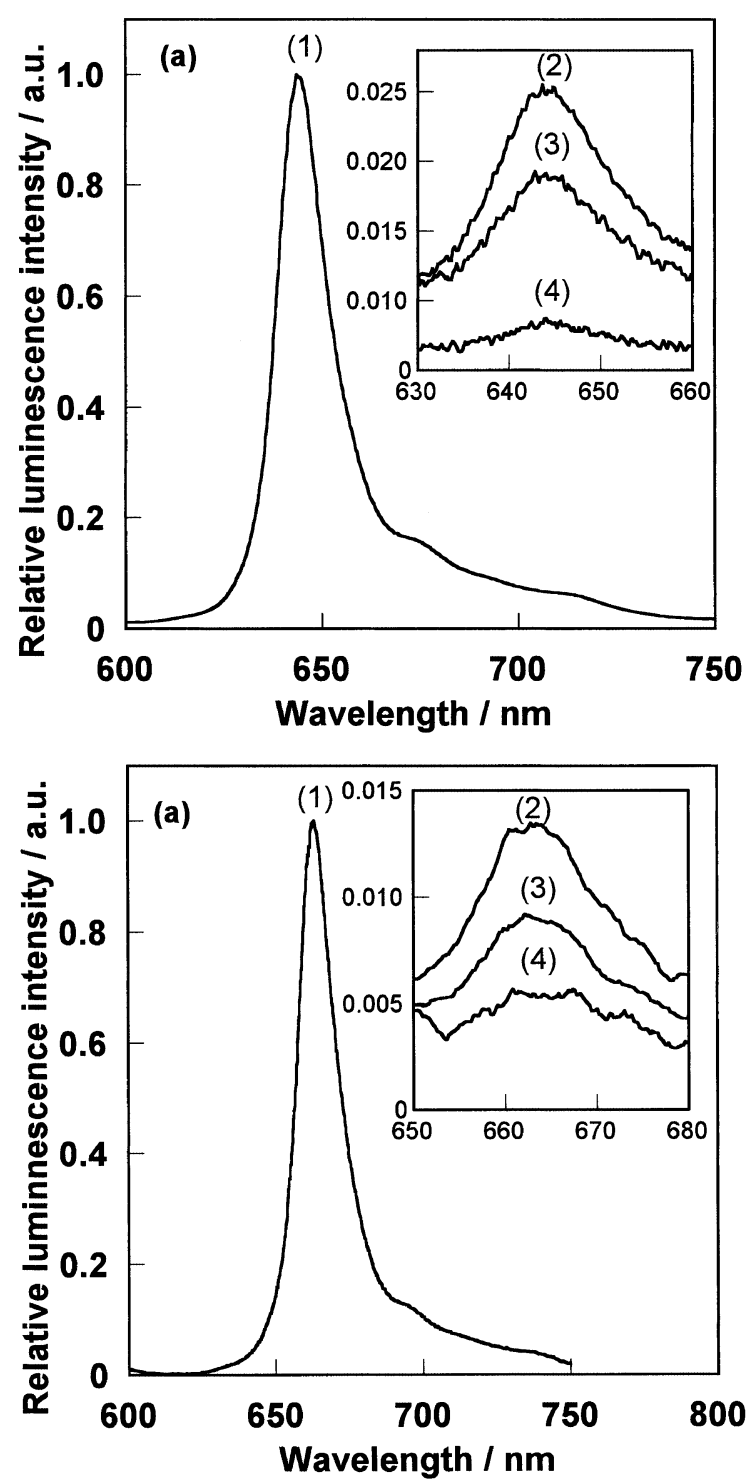

Figure 1. Luminescence spectra of PtOEP (a) and PdOEP (b) dispersed in the poly(TMSP) films, (1) $0 \%$; (2) $5.7 \%$; (3) $10 \%$, and (4) $100 \%$ oxygen. Excitation wavelengths for PtOEP and PdOEP were 535 and $546 \mathrm{~nm}$, respectively.

(absorption maxima $=534,501$, and $378 \mathrm{~nm}$ in the poly(TMSP) film; 535, 500, and $377 \mathrm{~nm}$ in toluene solution). On the other hand, the absorption spectrum of PdOEP in the poly(TMSP) film also was almost the same as in a solution (absorption maxima $=546$, 512 , and $394 \mathrm{~nm}$ in the poly(TMSP) film; 546, 512, and $393 \mathrm{~nm}$ in toluene solution). Thus, OEP metal complex molecules are homogeneously dispersed in the poly(TMSP) films. This denies any electrical interaction between OEP metal complex and poly(TMSP) in the ground state.

Luminescence Spectrum Change of OEP Metal Complex Dispersed in the Poly(TMSP) Film by Oxygen

OEP metal complex dispersed in the poly(TMSP) films showed luminescence at 646 and $664 \mathrm{~nm}$, respec-
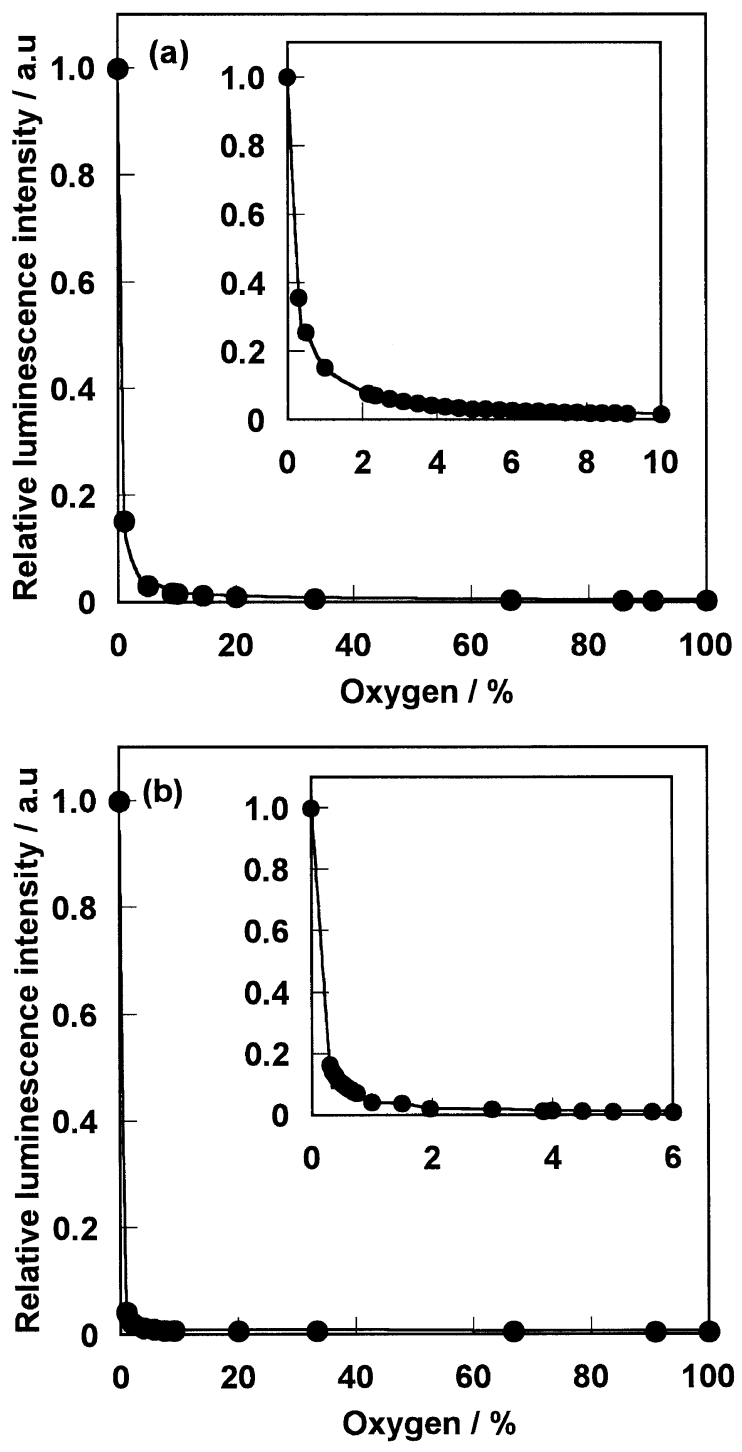

Figure 2. Relative luminescence intensity changes of PtOEP (a) and PdOEP (b) dispersed in the poly(TMSP) films under various oxygen concentrations. Excitation and emission wavelengths for PtOEP and PdOEP were 535 and $546 \mathrm{~nm}$, and 646 and $664 \mathrm{~nm}$, respectively. The inset shows the intensity changes in the oxygen concentration range between 0.3 and $10 \%$ for PtOEP and between 0.3 and $6.0 \%$ for PdOEP.

tively, when excited at a wavelength attributed to the Qband (535 nm for PtOEP and $546 \mathrm{~nm}$ for PdOEP). The luminescence spectral change of PtOEP (a) and PdOEP (b) in the poly(TMSP) film are shown in Figure 1. The luminescence intensity of the film depended on the oxygen concentration. These results indicate that the luminescence of PtOEP and PdOEP in the poly(TMSP) films was quenched by oxygen. The luminescence intensity of the PtOEP (a) and PdOEP (b) films decreased with an increase of oxygen concentration as shown in Figure 2. The ratio $I_{0} / I_{100}$ has been used as a sensitivity of the sensing film; the sensor having the ratio $I_{0} / I_{100}$ more than 3.0 is a suitable oxygen sensing device. ${ }^{33}$ The $I_{0} / I_{100}$ of PtOEP dispersed in the poly(TMSP), 
polystyrene and poly(DMS) films were $225,4.5$, and 5.5 , respectively. On the other hand, the $I_{0} / I_{100}$ of PdOEP dispersed in the poly(TMSP), polystyrene and poly(DMS) films were 121, 46.0, 50.1, respectively. These results indicate that PtOEP and PdOEP dispersed in the poly(TMSP) film is a potential candidate of highly sensitive devices for oxygen. Next let us focus on the limit of oxygen detection of OEP metal complex dispersed in the poly(TMSP) films. The $I_{0.3} / I_{0}$ values $\left(I_{0.3}\right.$ represents the detected luminescence intensity from a film exposed to $0.3 \%$ oxygen) of PtOEP dispersed in the poly(TMSP), polystyrene and poly(DMS) films are estimated to be $0.358,0.996$, and 0.990 , respectively. On the other hand, the $I_{0.3} / I_{0}$ values of PdOEP dispersed in the poly(TMSP), polystyrene and poly(DMS) films are estimated to be $0.164,0.797$, and 0.787 , respectively. For PtOEP and PdOEP dispersed in the poly(TMSP) films, the luminescence intensity was drastically changed by the oxygen concentrations, and the limit of oxygen detection was less than $0.3 \%$. This result indicates that OEP metal complex dispersed in the poly(TMSP) films are especially useful for sensing a dilute oxygen.

Stern-Volmer Relationship for OEP Metal Complex Dispersed in the Poly(TMSP) Film

Figure 3 shows the Stern-Volmer plots for PtOEP (a) and PdOEP (b) dispersed in the poly(TMSP) films. For PtOEP dispersed in the poly(TMSP) film, the plot exhibited considerable linearity in the low oxygen concentration range between 0 and $10 \%$ as shown in the inset of Figure 3a. At higher oxygen concentrations, on the other hand, Stern-Volmer plot showed nonlinear. Demas et al. reported a multi-site model that sensing film has some different oxygen-accessible site. ${ }^{34}$ According to this model, the site has own individual characteristic quenching constant. As the observed luminescence intensity was a sum of emission from different oxygen-accessible site with its own characteristic quenching constant, Stern-Volmer relationship is given by

$$
I_{0} / I=\left[\Sigma\left(f_{n} /\left(1+K_{S V n}\left[\mathrm{O}_{2}\right]\right)\right)\right]^{-1}
$$

where $n$ is an integer. $f_{n}$ is the fractional contributions to each oxygen accessible site. $K_{S V n}$ is the quenching constant for each accessible site. The best-fit curve was obtained when $n$ was equal to 2 by the eq 3. In Figure $3 \mathrm{a}$, the solid line is the best-fit using above equation $(n=2)$. The correlation factor of the plots, $r^{2}$, estimated to be 0.998 by the least squares method, indicating that the PtOEP dispersed in poly(TMSP) film sensor is calibrated by the modified Stern-Volmer equation. Thus, there are two oxygen accessible sites in
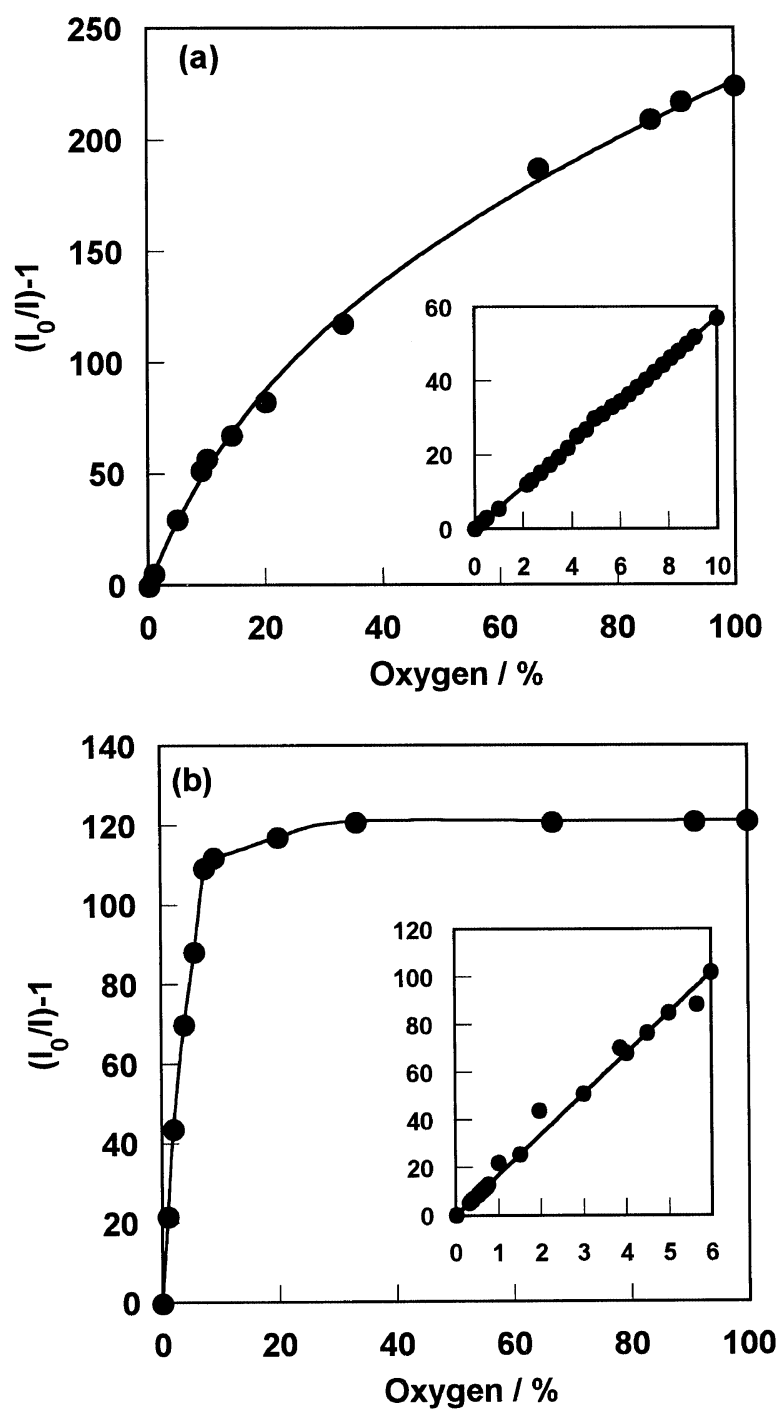

Figure 3. Stern-Volmer plots for PtOEP (a) and PdOEP (b) dispersed in the poly(TMSP) films. Excitation and emission wavelengths for PtOEP and PdOEP were 535 and $546 \mathrm{~nm}$, and 646 and $664 \mathrm{~nm}$, respectively. The inset shows the Stern-Volmer plots in the oxygen concentration range between 0.3 and $10 \%$ for PtOEP and between 0.3 and $6.0 \%$ for PdOEP. The solid lines are the bestfit curve using eq $3(n=2)$ in Figure (a).

the sensing films; one is an oxygen accessible $\left(K_{\mathrm{sv} 1}\right.$ $\left.=6.6 \%^{-1}, f_{1}=0.996\right)$ and the other is an oxygen difficult accessible site $\left(K_{\mathrm{sv} 2}=0.0042 \%^{-1}, f_{2}=0.004\right)$. The $K_{\mathrm{sv} 2}$ is very low value $\left(0.0042 \%^{-1}\right)$ and low contribution $\left(f_{2}=0.004\right)$ compared with $K_{\mathrm{sv} 1}$. Thus, the oxygen difficult accessible site is attributed to the static quenching site in film (emission from background scattering of glass slide substrate, etc.). The $K_{\mathrm{SV} 1}$ of PtOEP dispersed in the poly(TMSP) film was estimated to be $6.6 \%^{-1}$, which was much higher than those of the polystyrene film $\left(0.13 \%^{-1}\right)$ and the poly(DMS) film $\left(0.20 \%{ }^{-1}\right)$. This result indicates PtOEP dispersed in the poly(TMSP) film was higher sensitive device for oxygen. For PdOEP, on the other hand, the plot of dispersed in the poly(TMSP) film exhibits consider- 
able linearity at lower oxygen concentration range between 0 and $6.0 \%$, (the inset of Figure $3 \mathrm{~b}$ ), although the curvatures decrease at higher oxygen concentrations (Figure $3 b$ ). From Figures $2 b$ and $3 b$, the luminescence of PdOEP was effectively quenched by oxygen concentration up to $6.0 \%$. In general, the luminescence PdOEP was more quenchable than that of PtOEP by oxygen gas. ${ }^{27}$ This result suggested that the luminescence of PdOEP was almost fully quenched at the oxygen concentration of $c a .6 .0 \%$. The $K_{\mathrm{SV}}$ of PdOEP dispersed in the poly(TMSP) film was estimated to be $17.0 \%^{-1}$ in the oxygen concentration range between 0 and $6.0 \%$, which was much higher than those of the polystyrene film $\left(0.85 \%^{-1}\right)$ and the poly(DMS) film $\left(0.90 \%{ }^{-1}\right)$. This result indicates PdOEP dispersed in the poly(TMSP) film was higher sensitive device for trace oxygen. Oxygen permeability coefficients of poly(TMSP), polystyrene and poly(DMS) films at $30^{\circ} \mathrm{C}$ were reported to be 7700, 2.63, and 760 barrer (1 barrer $\left.=1 \times 10^{-10} \mathrm{~cm}^{3}(\mathrm{STP}) \mathrm{cm} \mathrm{cm}^{-2} \mathrm{~s} \mathrm{cmHg}\right)$, respectively. ${ }^{31,34-37}$ The oxygen permeability of poly(TMSP) is higher than those of polystyrene and poly(DMS). Such enormously high permeability has been, e.g., successfully applied to a film matrix for cobalt porphyrinmediated oxygen transport. ${ }^{38}$ Thus, higher sensitive optical sensor is developed using poly(TMSP) as a polymer matrix.

Operational Stability, and Response Time of OEP Metal Dispersed in the Poly(TMSP) Film

Figure 4 shows an operational stability test conducted by reading intensity signal from PtOEP (a) and PdOEP (b) dispersed in the poly(TMSP) films when oxygenated and deoxygenated gases were switched. The response times for optical oxygen sensor are defined the $95 \%$ response and recovery times, exhibited by the sensors when they are exposed to an alternating atmosphere of oxygen and argon, respectively. For PtOEP, the response times of the film are $3.6 \mathrm{~s}$ on going from argon to oxygen and $73.2 \mathrm{~s}$ on going from oxygen to argon, respectively (for PtOEP dispersed in polystyrene and in the poly(DMS) films, 35.0 and $10.0 \mathrm{~s}$ on going from argon to oxygen and 100 and $82.0 \mathrm{~s}$ on going from oxygen to argon, respectively). For PdOEP, on the other hand, the response times of the film are $3.2 \mathrm{~s}$ on going from argon to oxygen and $250 \mathrm{~s}$ on going from oxygen to argon, respectively (for PdOEP dispersed in polystyrene and in the poly(DMS) films, 10.6 and $9.0 \mathrm{~s}$ on going from argon to oxygen and 158.8 and $200.2 \mathrm{~s}$ on going from oxygen to argon, respectively). By using poly(TMSP) as polymer matrix, the fast response time was obtained. The signal changes were fully reversible and measurement hyster-
(1)

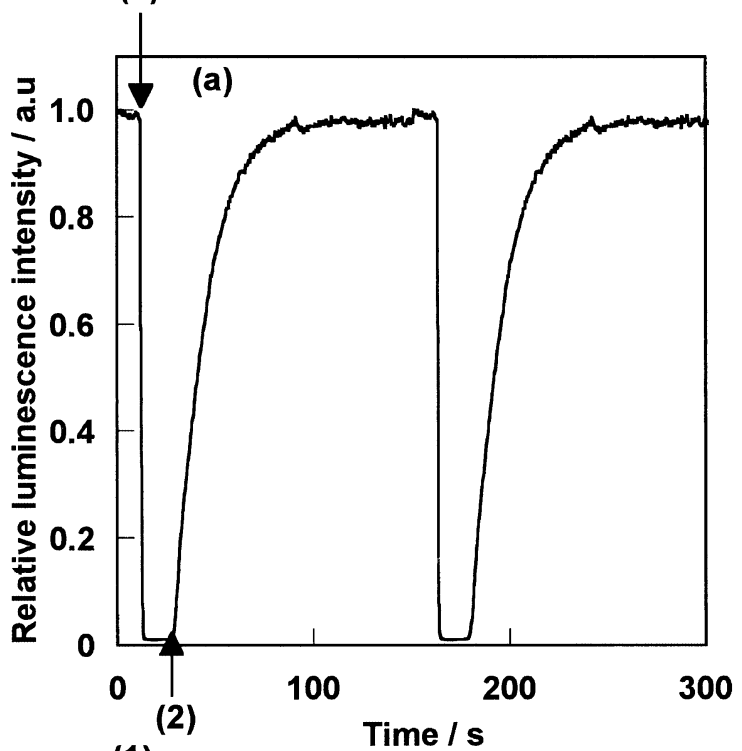

(1)

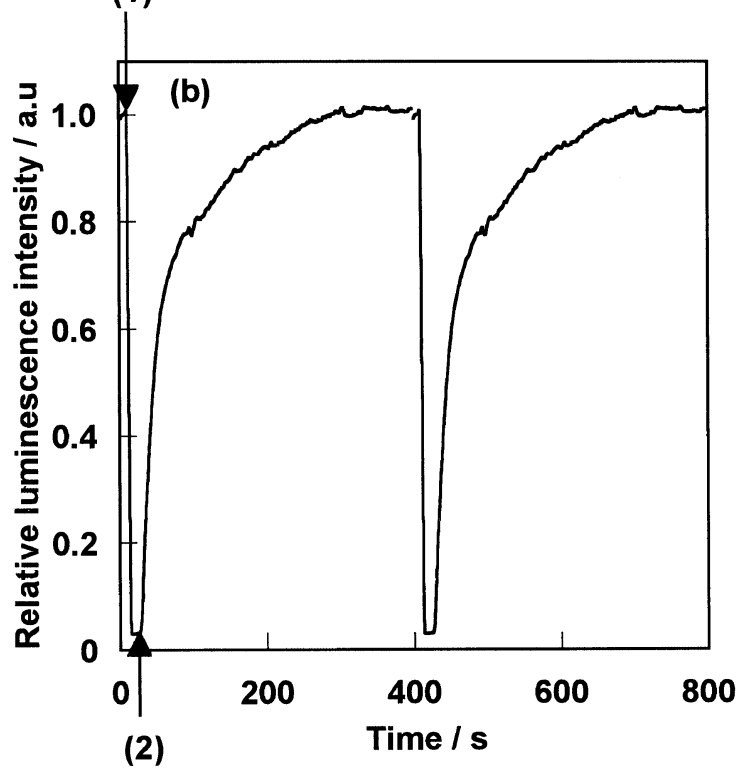

Figure 4. Response time and relative intensity change for PtOEP (a) and PdOEP (b) dispersed in the poly(TMSP) films on switching between $100 \%$ argon (1) and $100 \%$ oxygen (2). Excitation wavelengths for PtOEP and PdOEP were 535 and $546 \mathrm{~nm}$, respectively. Emission wavelengths for PtOEP and PdOEP were 646 and $664 \mathrm{~nm}$, respectively.

isis was not observed.

Dynamic response of PtOEP (a) and PdOEP (b) dispersed in the poly(TMSP) films under various oxygen concentrations is shown in Figure 5. This test was repeated and signal changes were monitored when increasing and decreasing oxygen concentrations change randomly. In the cases of both OEP metal complexes, evidently, the signal changes were fully reversible and measurement hysterisis was not observed.

In general, oxygen sensors using dye dispersed in polymer film are strongly affected by the thickness of the film. A thinner film requires less time for endoge- 

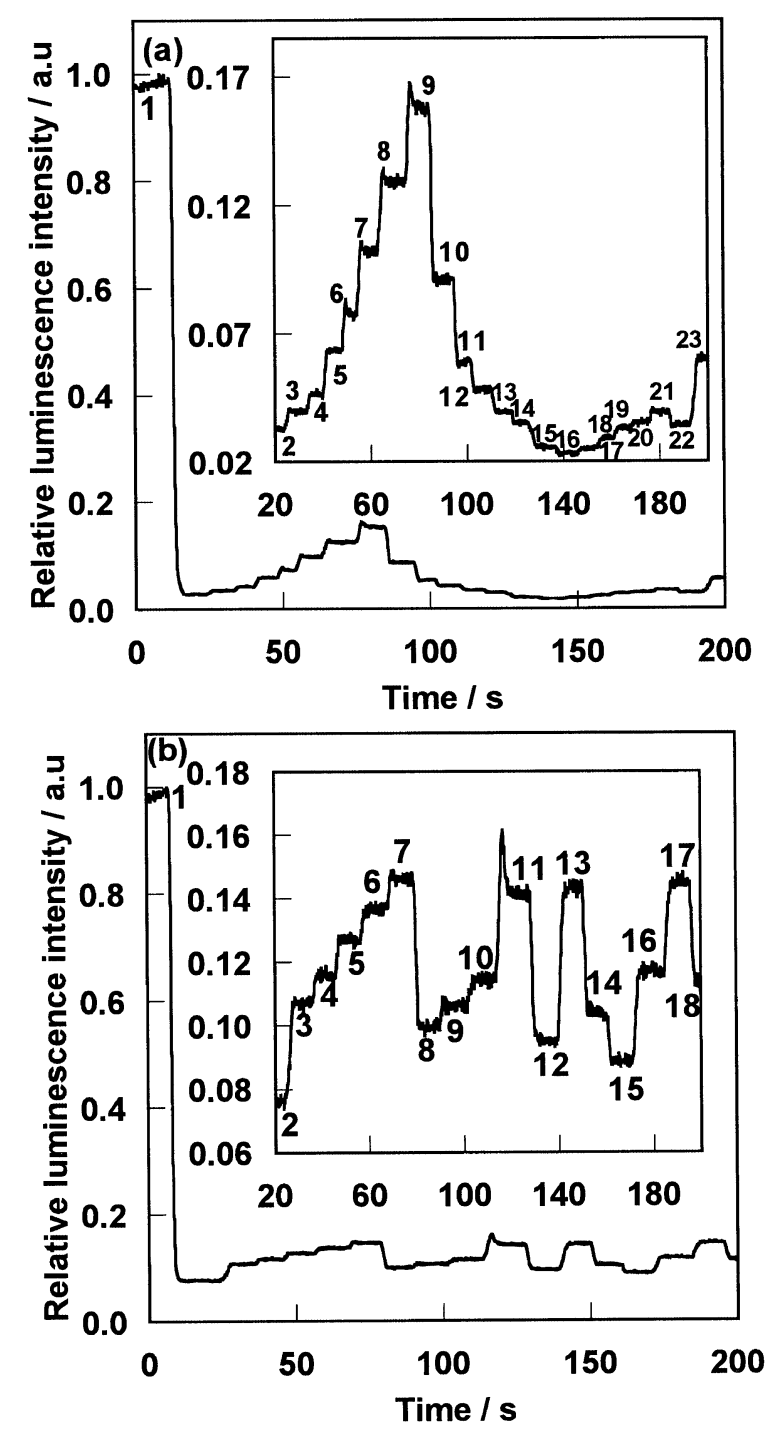

Figure 5. Dynamic response of PtOEP (a) and PdOEP (b) dispersed in poly(TMSP) films when oxygen concentrations were changed randomly. For PtOEP film: (1) 0 ; (2) 5.5 ; (3) 5.0 ; (4) 3.8 ; (5) 2.7 ; (6) 2.1 ; (7) 1.6 ; (8) 1.2 ; (9) 1.0 ; (10) 1.9 ; (11) 3.0 ; (12) 3.8 ; (13) 5.0 ; (14) 5.5 ; (15) 8.6; (16) 10; (17) 6.3 ; (18) 6.3; (19) 6.2 ; (20) 5.5 ; (21) 5.5 ; (22) 6.2 ; (23) $3.0 \%$ oxygen. For PdOEP film: (1) 0 ; (2) 0.71 ; (3) 0.49 ; (4) 0.45 ; (5) 0.41 ; (6) 0.34 ; (7) 0.38 ; (8) 0.54 ; (9) 0.49 ; (10) 0.45 ; (11) 0.36 ; (12) 0.56 ; (13) 0.36 ; (14) 0.49 ; (15) 0.34 ; (16) 0.45 ; (17) 0.34 ; (18) $0.45 \%$ oxygen. Excitation wavelengths for PtOEP and PdOEP were 535 and $546 \mathrm{~nm}$, respectively. Emission wavelengths for PtOEP and PdOEP were 646 and 664 $\mathrm{nm}$, respectively.

nous oxygen migration to reach equilibrium with the external environment. For PtOEP and PdOEP dispersed in the poly(TMSP) films, however, $K_{\mathrm{SV}}$ was little affected by differences of 10 and $50 \mu \mathrm{m}$ in the film thickness. An important factor for application of the OEP metal complex dispersed in the poly(TMSP) films as an optical oxygen sensing material, is its photostability. To characterize the photostability of the OEP metal complex dispersed in the poly(TMSP) film, the absorption spectrum of the film was measured after continuous ir- radiation using a $150 \mathrm{~W}$ tungsten lamp on the film for $12 \mathrm{~h}$. After irradiation for $12 \mathrm{~h}, 3.0$ and $3.5 \%$ decreases of initial absorption intensity of PtOEP at $378 \mathrm{~nm}$ and PdOEP at $390 \mathrm{~nm}$ were observed, indicating that the OEP metal complex dispersed in the poly(TMSP) films are stable against irradiation.

\section{CONCLUSIONS}

In this paper, PtOEP and PdOEP dispersed in the highly gas permeable polymer, poly(TMSP) film, is reported. The $I_{0} / I_{100}$ values of PtOEP and PdOEP in the poly(TMSP) film is estimated to be 225 and 121 and large Stern-Volmer constants $\left(K_{\mathrm{SV}}=6.6 \%^{-1}\right.$ for PtOEP and $17.0 \%^{-1}$ for PdOEP) are obtained compared with the PtOEP or PdOEP dispersed in poly(DMS) and polystyrene films. Especially, the luminescence intensity of PdOEP dispersed in the poly(TMSP) film was drastically changed by the oxygen concentrations. The limits of oxygen detection of PtOEP and PdOEP dispersed in the poly(TMSP) films was less than $0.3 \%$. This result indicates that PtOEP and PdOEP dispersed in the poly(TMSP) films are useful sensing device for a trace analysis of oxygen. As the oxygen permeability of the poly(TMSP) film is not lost at a lower temperature about $-196^{\circ} \mathrm{C}$, the oxygen sensing performance of OEP metal complex dispersed in the poly(TMSP) films at cryogenic temperature (around the liquefaction temperature of oxygen; $-183^{\circ} \mathrm{C}$ ) is now being studied.

Acknowledgment. This work is partially supported by "Molecular Sensors for Aero-Thermodynamic Research (MOSAIC)", the Special Coordination Funds from the Ministry of Education, Culture, Sports, Science and Technology Agency.

\section{REFERENCES}

1. C. Prininger, I. Klimant, and O. S. Wolfbeis, Anal. Chem., 66, 1841 (1994).

2. R. C. Martin, S. F. Malin, D. J. Bartnil, A. M. Schilling, and S. C. Furlong, Proc. SPIE., 2131, 426 (1994).

3. M. J. Atkinson, F. I. M. Thomas, N. Larson, E. Terrill, K. Morita, and C. C. Lium, Deep-Sea Res. I., 42, 761 (1995).

4. D. A. Skoog, D. M. West, and F. J. Holler, "Fundamentals of Analytical Chemistry", Saunders, Philadelphia, PA, 1988, p 344.

5. L. C. Clark, Trans. Am. Soc. Artif. Intern. Organs, 2, 41 (1956).

6. T. M. Freeman and W. R. Seitz, Anal. Chem., 53, 98 (1981).

7. H. D. Hendricks, U. S. Patent 3709663 (Jan. 9, 1973).

8. Y. Suzuki, H. Nishide, and E. Tsuchida, Macromolecules, 33, 2530 (2000).

9. T. Ishiji and M. Kaneko, Analyst, 120, 1633 (1995). 
10. A. Sharma and O. S. Wolfbeis, Appl. Spect., 42, 1009 (1988).

11. E. D. Lee, T. C. Werner, and R. Seitz, Anal. Chem., 59, 279 (1987).

12. S. M. Ramasamy and R. J. Hurubise, Anal. Chim. Acta, 152, 83 (1983).

13. H. W. Kroneis and H. J. Marsoner, Sens. Actuators, 4, 587 (1983).

14. W. Xu, R. Schmidt, M. Whaley, J. N. Demas, B. A. DeGraff, E. K. Karikari, and B. L. Farmer, Anal. Chem., 67, 3172 (1995).

15. J. Olmsted, Chem. Phys. Lett., 26, 33 (1974).

16. P. Hartmann, M. J. P. Leiner, and M. E. Lippitsch, Anal. Chem., 67, 88 (1995).

17. M. G. Sasso, F. H. Quina, and E. J. H. Bechera, Anal. Biochem., 156, 239 (1986).

18. E. Singer, G. L. Duveneck, M. Ehrat, and M. Widmer, Sens. Actuators, A, 41, 542 (1994).

19. E. R. Carraway, J. N. Demas, B. A. DeGraff, and J. R. Bacon, Anal. Chem., 63, 332 (1991).

20. J. R. Bacon and J. N. Demas, Anal. Chem., 59, 2780 (1987).

21. X. M. Li and H. Y. Wong, Anal. Chim. Acta, 262, 27 (1992).

22. W. Y. Xu, K. A. Kneas, J. N. Demas, and B. A. DeGraff, Anal. Chem., 68, 2605 (1996).

23. L. Sacksteder, J. N. Demas, and B. A. DeGraff, Anal. Chem., 65, 3480 (1993).

24. D. B. Papkovsky, G. V. Ponomarev, W. Trettnak, and P. O'Leary, Anal. Chem., 67, 4112 (1995).

25. J. Vanderkooi, G. Maniara, J. Green, and D. F. Wilson, J. Biol.
Chem., 262, 5476 (1987).

26. Mills and A. Lepre, Anal. Chem., 69, 4653 (1997).

27. K. Kalyanasundaram, "Photochemistry of Polypyridine and Porphyrin Complexes", Academic Press, Inc., New York, N.Y., 1992, p 500.

28. S-K. Lee and I. Okura, Anal. Sci., 13, 535 (1997).

29. S-K. Lee and I. Okura, Spectrochim. Acta, Part A, 54, 91 (1998).

30. S. Pauly, "Polymer Handbook, 3rd ed.", John Wiley \& Sons, Inc., New York, N.Y., 1989.

31. T. Masuda, E. Isobe, T. Higashimura, and K. Takada, J. Am. Chem. Soc., 105, 7473 (1983).

32. B. D. MacCraith, C. M. McDonagh, G. O'Keeffe, E. T. Keyes, J. G. Vos, B. O'Kelly, and J. F. McGilp, Analyst, 118, 385 (1993).

33. J. N. Demas, B. A. DeGraff, and W. Xu, Anal. Chem., 67, 1377 (1995).

34. T. Masuda, E. Isobe, and T. Higashimura, Macromolecules, 18, 841 (1985).

35. K. Takada, H. Matsuya, T. Masuda, and T. Higashimura, J. Appl. Polym. Sci., 30, 1605 (1985).

36. T. Masuda, Y. Iguchi, B-Z. Tang, and T. Higashimura, Polymer, 29, 2041 (1988).

37. Y. Ichiraku, S. A. Stern, and T. Nakagawa, J. Membr. Sci., 34, 5 (1987).

38. H. Nishide, H. Kawakami, Y. Sasame, K. Ishikawa, and E. Tsuchida, J. Polym. Sci., Part A: Polym. Chem., 30, 77 (1992). 